\title{
Article \\ Comparative Genomic Analysis of Bifidobacterium bifidum Strains Isolated from Different Niches
}

\author{
Wenwei Lu ${ }^{1,2,3}$, Zhangming Pei ${ }^{1,2}{ }^{\mathbb{D}}$, Mengning Zang ${ }^{1,2}$, Yuan-kun Lee ${ }^{4} \mathbb{D}_{\text {, Jianxin Zhao }}{ }^{1,2}$, Wei Chen ${ }^{1,2,3}$, \\ Hongchao Wang ${ }^{1,2, *(\mathbb{D})}$ and Hao Zhang $1,2,3,5, *$ (D)
}

1 State Key Laboratory of Food Science and Technology, Jiangnan University, Wuxi 214122, China; luwenwei@jiangnan.edu.cn (W.L.); 7190112086@stu.jiangnan.edu.cn (Z.P.); 6190111125@stu.jiangnan.edu.cn (M.Z.); jianxinzhao@jiangnan.edu.cn (J.Z.); chenwei66@jiangnan.edu.cn (W.C.)

2 School of Food Science and Technology, Jiangnan University, Wuxi 214122, China

3 National Engineering Research Center for Functional Food, Jiangnan University, Wuxi 214122, China

4 Department of Microbiology \& Immunology, Yong Loo Lin School of Medicine, National University of Singapore, Singapore 117597, Singapore; micleeyk@nus.edu.sg

5 Wuxi Translational Medicine Research Center and Jiangsu Translational Medicine Research Institute Wuxi Branch, Wuxi 214122, China

* Correspondence: hcwang@jiangnan.edu.cn (H.W.); zhangzhao@jiangnan.edu.cn (H.Z.); Tel.: +86-510-85-197-239 (H.W. \& H.Z.); Fax: +86-510-85-197-239 (H.W. \& H.Z.)

Citation: Lu, W.; Pei, Z.; Zang, M.; Lee, Y.-k.; Zhao, J.; Chen, W.; Wang, H.; Zhang, H. Comparative Genomic Analysis of Bifidobacterium bifidum Strains Isolated from Different Niches. Genes 2021, 12, 1504. https:/ / doi.org/ 10.3390/genes12101504

Academic Editor:

Alfonso Benítez-Páez

Received: 7 July 2021

Accepted: 17 September 2021

Published: 25 September 2021

Publisher's Note: MDPI stays neutral with regard to jurisdictional claims in published maps and institutional affiliations.

Copyright: (c) 2021 by the authors. Licensee MDPI, Basel, Switzerland. This article is an open access article distributed under the terms and conditions of the Creative Commons Attribution (CC BY) license (https:/ / creativecommons.org/licenses/by/ $4.0 /)$.

\begin{abstract}
The potential probiotic benefits of Bifidobacterium bifidum have received increasing attention recently. We used comparative genomic analysis to explore the differences in the genome and the physiological characteristics of B. bifidum isolated from the fecal samples of Chinese adults and infants. The relationships between genotypes and phenotypes were analyzed to assess the effects of isolation sources on the genetic variation of B. bifidum. The phylogenetic tree results indicated that the phylogeny of B. bifidum may be related to the geographical features of its isolation source. B. bifidum was found to have an open pan-genome and a conserved core genome. The genetic diversity of $B$. bifidum is mainly reflected in carbohydrate metabolism- and immune/competition-related factors, such as the glycoside hydrolase gene family, bacteriocin operons, antibiotic resistance genes, and clustered regularly interspaced short palindromic repeats (CRISPR)-Cas. Additionally, the type III A CRISPR-Cas system was discovered in B. bifidum for the first time. B. bifidum strains exhibited niche-specific characteristics, and the results of this study provide an improved understanding of the genetics of this species.
\end{abstract}

Keywords: Bifidobacterium bifidum; comparative genomics; diversity; genotype

\section{Introduction}

Bifidobacterium bifidum is a bifidobacterial species with a high prevalence in the human gut microbiome [1]. It is among the earliest bacterial species to colonize the intestinal tract of newborns [2]. It is generally believed that B. bifidum present in the intestines of infants may originate from vertical maternal transmission [3], and its predominance in the intestinal tract of breastfed infants is due to its capacity to utilize specific host-derived glycans, such as human milk oligosaccharides [4,5] and mucin [6]. The beneficial effects of B. bifidum include restoring injured intestinal mucosa [7], modulating perturbation and the gut metabolic profile [8], suppressing colitis [9], reducing cholesterol levels [10], and ameliorating spatial memory impairment [11]. Therefore, B. bifidum has powerful probiotic functions and the potential to prevent or treat a range of human diseases. It is currently available as a functional food ingredient, and it can also be exploited as a therapeutic [12].

In recent years, the genomes of many bifidobacterial strains, especially those used in the probiotic industry, have been sequenced [13]. As of December 2020, more than $100 B$. bifidum genome assemblies have been deposited in the National Center for Biotechnology 
Information (NCBI) GenBank database, and approximately two-thirds of these were newly sequenced in the past three years, indicating that B. bifidum genomics is a hot research topic. Turroni et al. [6] studied the mechanism of mucin metabolism in B. bifidum PRL2010 and found that a set of chromosomal loci that encode specific mucin-degrading enzymes is prevalent in B. bifidum. Kim et al. [14] evaluated the safety of B. bifidum BGN4, in terms of genome stability, antibiotic resistance gene (ARG) transferability, and the presence of virulence factors. Zhurina et al. completely annotated the genome sequence of $B$. bifidum S17, which was shown to strongly adhere to intestinal epithelial cells and has potent anti-inflammatory activity in vitro and in vivo [15]. Gueimonde et al. reported the genome sequences of B. bifidum LMG13195, which can interact with human immune cells, generating functional regulatory $\mathrm{T}$ cells [16]. Andryuschenko et al. described the draft genome sequence of $B$. bifidum ICIS-310, isolated from the feces of a healthy 5-year-old child from Orenburg [17].

With an increasing number of available genomes for a single species, comparative genomics techniques are frequently used to analyze the genetic background, habitat adaptability, and evolutionary changes of bacterial species. Many comparative genomics studies have been published for common bifidobacterial species, such as B. longum, B. breve, $B$. animalis, and B. adolescentis [18-21]. Several studies have depicted the genetic diversity of B. bifidum strains using random amplified polymorphic DNA (RAPD) [22] and multilocus sequence typing (MLST) [23]. In contrast, there is only one comparative genomics study of B. bifidum [13]. In that study, Duranti et al. sequenced the genomes of 15 B. bifidum strains and proposed that $B$. bifidum harbors a closed pan-genome and a conserved core genome. The ecological adaptability of $B$. bifidum was also noted, based on its utilization of host-derived glycans [13]. However, we propose that these findings need to be verified by analyzing additional $B$. bifidum genomes. Furthermore, the B. bifidum genomes that have been sequenced thus far are predominantly from Europe or the USA. To systematically analyze the genomic diversity and phylogenetic evolution of B. bifidum, and to extend our knowledge of other aspects of this species, it is crucial to expand the dataset with strains isolated from different regions.

In this study, we isolated 85 B. bifidum strains from the fecal samples of Chinese adults or infants. We sequenced the genomes of these strains and assembled them into scaffolds. The average nucleotide identity (ANI) was then calculated, the pan-genome and core genome were analyzed, and a phylogenetic tree was constructed for $140 \mathrm{~B}$. bifidum genomes (including 55 reference assembly sequences obtained from GenBank). These data provided a more detailed and reliable basis for assessing the genome openness and genetic diversity of $B$. bifidum. We then identified the CRISPR-Cas systems, prophage fragments, and bacteriocin genes in all 140 B. bifidum genomes, resulting in invaluable information about the genome composition, mobile elements, and defense systems of the species. Finally, a comprehensive scanning of carbohydrate-active enzymes (CAZys) and ARGs was performed. Experiments investigating the carbohydrate utilization capacity and antibiotic resistance of the 85 isolated strains were performed to identify potential genotype-phenotype associations in B. bifidum.

\section{Materials and Methods}

\subsection{Bacterial Strain Screening}

The fecal samples from Chinese adults and infants used in this study were previously collected by our team and were randomly selected for strain screening. After appropriate serial dilution, each sample was spread on DeMan-Rogosa-Sharpe (MRS) agar culture medium supplemented with $0.05 \%(w / v)$ L-cysteine hydrochloride and incubated at $37{ }^{\circ} \mathrm{C}$ for $24-48 \mathrm{~h}$ in a chamber with an anaerobic atmosphere $\left(10 \%[v / v] \mathrm{H}_{2}, 10 \%[v / v] \mathrm{CO}_{2}\right.$ and $80 \%[v / v] \mathrm{N}_{2}$ ). Several colonies were picked from each plate and streaked on MRS medium to obtain pure colonies. The final pure culture was incubated at $37^{\circ} \mathrm{C}$ for $24 \mathrm{~h}$ under anaerobic conditions, and preserved in $30 \%(w / v)$ glycerol at $-80{ }^{\circ} \mathrm{C}$. The species of all strains were preliminarily determined using $16 \mathrm{~S}$ rRNA-based identification (GENEWIZ Co., 
Ltd., Suzhou, China). All reagents used in the processes described above were purchased from Sinopharm Chemical Reagent Co., Ltd. (Shanghai, China).

\subsection{DNA Extraction, Genome Sequencing, Assembly, and Annotation}

All B. bifidum strains were cultured in liquid MRS medium and incubated at $37^{\circ} \mathrm{C}$ for 24-48 h. The bacterial culture medium was centrifuged at $6000 \times \mathrm{g}$ rpm for $3 \mathrm{~min}$, and then the supernatant was discarded. Next, bacterial cells were washed in $0.9 \%$ sterile normal saline and collected by centrifugation $(6000 \times \mathrm{g} \mathrm{rpm}, 3 \mathrm{~min})$. The rapid bacterial genomic DNA isolation kit (Sangon Biotech Ltd., Shanghai, China) was used for genomic DNA extraction.

B. bifidum genomes were sequenced at Novogene BioTech Co. (Tianjin, China) or Majorbio BioTech Co., Ltd. (Shanghai, China) using an Illumina Hiseq X Ten platform (San Diego, CA, USA). DNA samples that passed quality control were used for library construction, which yielded fragments of approximately $400 \mathrm{bp}$ for paired-end sequencing. Single-end sequencing yielded a read length of $150 \mathrm{bp}$, and raw data from each sample had no less than 100-fold genomic coverage. Sequence reads were assembled using SOAPdenovo v2.04 software [24], and the optimized sequence is spliced under multiple Kmer parameters to obtain the optimal assembly result. Local inner gaps filling and base correction were performed using the software GapCloser v1.12 [25]. CheckM (https: / / ecogenomics.github.io/CheckM/, accessed on 30 May 2020) [26] was used to evaluate the quality of assembly genomes. Protein-encoding open reading frames (ORFs) were predicted using Glimmer 3.02 [27]. Functional assignments were performed and manually edited based on similarity searches against a nonredundant protein database provided by the NCBI and the Cluster of Orthologous Groups protein database. Specific information on the strains and the quality data of each genome (genome size, GC content, genome level, number of scaffolds, and $\mathrm{N}_{50}$ value) are listed in Table S1.

\subsection{ANI Calculation, Pan-/Core Genome Analysis, Orthologous Gene Clustering, and Phylogenetic Tree Construction}

The pairwise ANI values of B. bifidum genomes were calculated and visualized using the PYANI v0.2.9 package (https:/ / github.com/widdowquinn/pyani/, accessed on 15 November 2020). Pan-genome and core-genome profiles were constructed using the PGAP v1.2.1 package [28], and all genes were divided into the core genome and the dispensable genome. Orthologous gene clustering and Venn diagram construction were performed using ORTHOMCL v2.0.9 [29]. All parameters were kept as default.

The orthologous genes of all B. bifidum genomes were aligned using MAFFT v7.3 [30]. Phylogeny Inference Package v3.6 was used to construct the phylogenetic tree, based on the aligned orthologous genes, using the neighbor-joining method. The final phylogenetic tree was visualized using the webserver EvolView v3 [31].

\subsection{Prediction of the CRISPR-Cas System, Prophages, and Bacteriocin Operons}

CRISPR arrays and repeat sequences were identified using the CRISPRCasFinder program (https:/ / crisprcas.i2bc.paris-saclay.fr/CrisprCasFinder/Index accessed on 15 November 2020) [32]. MacSyFinder [33] was used to scan the corresponding Cas genes and determine the type/subtype of the CRISPR-Cas system [33]. The secondary structure of CRISPR repeats was predicted using the RNAfold web server (http://rna.tbi.univie.ac.at//cgibin/RNAWebSuite/RNAfold.cgi/, accessed on 20 December 2020) [34]. The phylogenetic tree, based on the CRISPR repeats, was established using MEGA X [35]. PHASTER (http: / / phaster.ca/, accessed on 2 October 2020) [36] was used to identify prophage fragments in the B. bifidum genomes. Bacteriocin operons were identified and visualized using the BAGEL4 web server (http:/ / bagel4.molgenrug.nl/index.php/, accessed on 5 November 2020) [37]. All the above predictions were performed with default parameters supplied by the websites. 


\subsection{CAZy Scanning and Determination of Carbohydrate Utilization Capacity}

The genomes of all 85 isolated B. bifidum strains were aligned against the CAZy database (http:/ / www.cazy.org/, accessed on 25 November 2020) [38], and a conservative threshold (amino acid identity $\geq 30 \%$, E-value $\leq 1 \times 10^{-5}$ ) was used to predict putative CAZys. CAZys that were identified in this study included glycoside hydrolases (GHs), glycosyltransferases (GTs), carbohydrate esterases (CEs), and carbohydrate-binding modules (CBMs).

All 85 B. bifidum strains were precultured anaerobically in MRS liquid medium at $37^{\circ} \mathrm{C}$ for $24 \mathrm{~h}$. Bacterial cells were then collected by centrifugation at $8000 \times g$ for $3 \mathrm{~min}$, washed, and resuspended in sterile normal saline. All strains were inoculated with $2 \%(v / v)$ inoculum, into a series of MRS liquid media in which different carbohydrates were used as the sole carbon source. An MRS liquid medium with glucose and a carbohydrate-free liquid medium was used as the positive and negative controls, respectively. Carbohydrate utilization capacity tests were performed to evaluate seven carbohydrate sources, including lactose, 2'-fucosyllactose, mucin, sucrose, fructo-oligosaccharide, inulin, and soluble starch. A bromocresol purple solution $(0.5 \%, w / v)$ was added to the medium at $1.5 \%(v / v)$ as an indicator, and growth status was determined based on the color change in the medium. A change from purple (unavailable) to yellow (available) indicated the growth and acid production levels of the strain.

\subsection{ARG Prediction and Antibiotic Susceptibility Testing}

B. bifidum genomes were aligned against sequences from the latest version of the Comprehensive Antibiotic Resistance Database [39], and a conservative threshold (amino acid identity $\geq 30 \%$, comparison hit-bit score $\geq 37.0$ ) was used to predict putative ARGs.

The antibiotic susceptibility of the B. bifidum strains was evaluated using the broth microdilution method, according to ISO 10932:2010 [40]. The following 10 antibiotics were tested: tetracycline, erythromycin, clindamycin, ampicillin, amoxicillin, trimethoprim, ciprofloxacin, chloramphenicol, rifampicin, and vancomycin (all purchased from Sangon Biotech Co., Ltd., Shanghai, China). The microbiological breakpoints of Bifidobacterium recommended by the European Food Safety Authority were used to distinguish susceptible strains from resistant strains.

\subsection{Data Visualization and Statistical Analysis}

Line charts, violin plots, and histograms were constructed using Prism v8.0 (GraphPad, San Diego, CA, USA). The heatmaps of CAZys, carbohydrate utilization capacity, and ARGs were constructed using HemI v1.0 [41]. A Mann-Whitney U test was performed using SPSS PASW Statistics v18.0 (IBM, Armonk, NY, USA).

\subsection{Data Availability}

All 85 B. bifidum genomes sequenced in this study have been deposited in the NCBI GenBank database under project no. PRJNA681061. The accession numbers of all 140 sequences (including 55 reference B. bifidum genome sequences downloaded from GenBank) are listed in Table S1.

\section{Results}

\subsection{General Features and ANI Values of B. bifidum}

We obtained more than 100 B. bifidum isolates from 85 samples in this study. One strain from each independent sample was then selected for draft-genome sequencing. Of these 85 B. bifidum strains, $68 \%(58 / 85)$ were isolated from infant feces, and the other 27 were isolated from adult feces. The genomes of these $85 \mathrm{~B}$. bifidum strains were subjected to further analysis, along with another 55 publicly available B. bifidum reference genomes. Specific information on all 140 strains/genomes is given in Table S1. The genome sizes of most B. bifidum strains were $2.03-2.55 \mathrm{Mb}$, with an average of $2.17 \pm 0.09 \mathrm{Mb}$, and each genome contained an average of $1837 \pm 143$ ORFs. However, a unique sequence was 
identified in B. bifidum 62_13, which had a genome size of only $1.68 \mathrm{Mb}$, which encoded the smallest number of ORFs $(1,365)$. The guanine and cytosine content $(\mathrm{G}+\mathrm{C}$ content) of B. bifidum was relatively stable, ranging from $62.3 \%$ to $62.8 \%$. The pairwise ANI values of B. bifidum genomes ranged from $97.73 \%$ to $99.99 \%$ (Figure 1), which was greater than the threshold of $96 \%$. This verified that all 140 strains, including B. bifidum 62_13, were the same species, without any subspecies. Moreover, the relatively high ANI values indicated only minor differences between B. bifidum genomes. The ANI threshold for differentiating strains within the same species was updated to $96 \%$ [42]. Due to the small difference between our inter-cluster ANI and the accepted threshold, we speculated that there are minor differences between B. bifidum genomes.

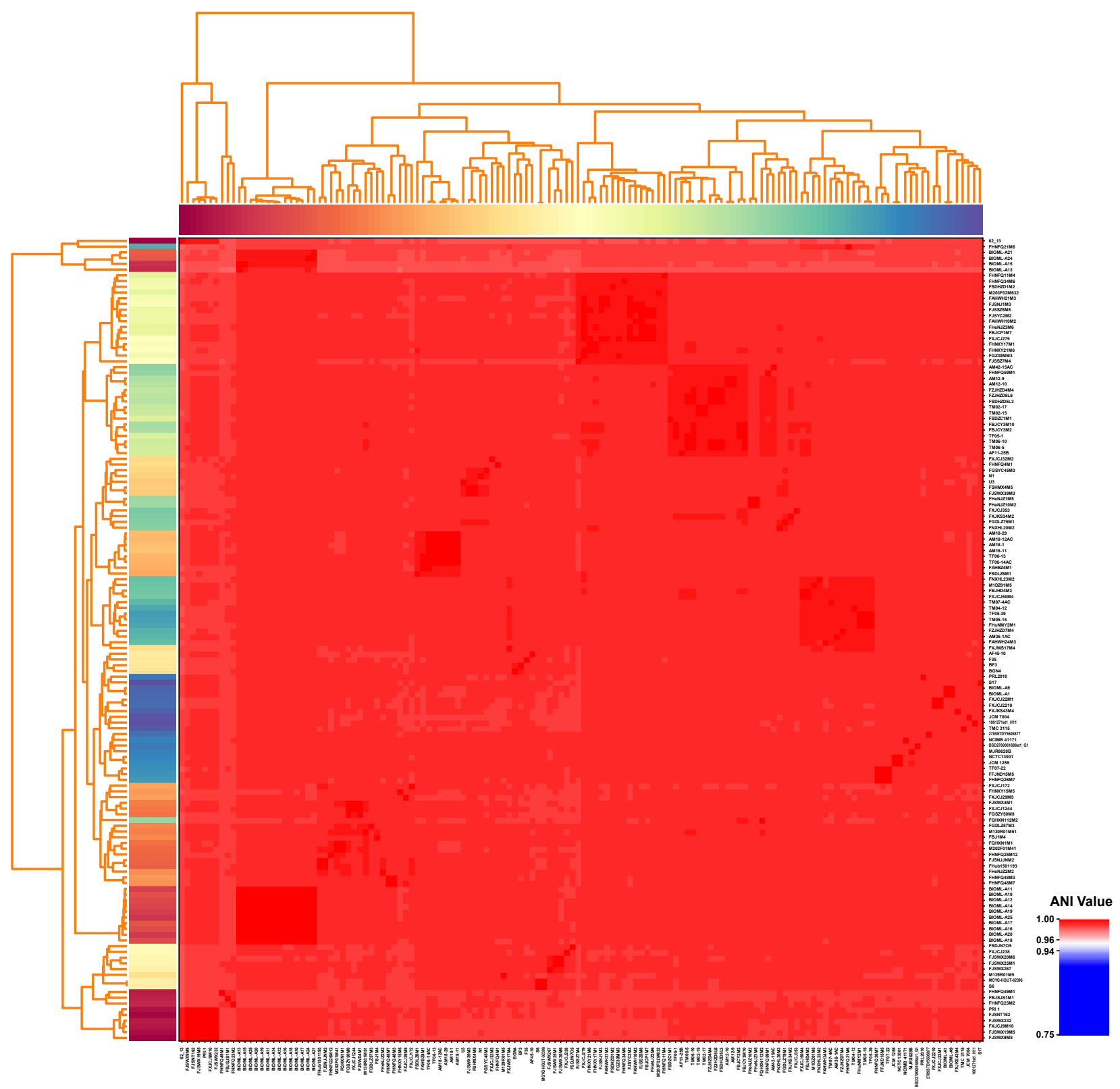

Figure 1. Pairwise average nucleotide identity values across 140 B. bifidum genomes. The color coding for the genomes on the $x$-axis and $y$-axis was used to differentiate the strains. 


\subsection{Pan-Genome and Core Genome of B. bifidum}

To determine the total number of different genes and the number of conserved genes present in representative $B$. bifidum strains, we performed pan-genome and core genome analyses of the $140 \mathrm{~B}$. bifidum genomes. The number of core genes and pan-genes and the number of sequenced strains were used to construct a functional relationship diagram. The results showed that the pan-genome of all 140 strains of B. bifidum consisted of 8399 genes. The pan-genome asymptotic curve did not reach a plateau (Figure 2A), suggesting that when more B. bifidum genomes are identified with novel genes, the pan-genome would continuously increase. Meanwhile, the exponential value of the deduced mathematical function was $>0.5$ (Figure 2A). These findings indicate that B. bifidum had an open pangenome. In contrast, the core genome demonstrated a power trend line that plateaued. This was represented by 638 genes and accounted for approximately $35 \%$ of the total number of gene families. The Venn diagram showed the presence of 683 homologous genes among all 140 B. bifidum genomes, while the specific number of genes for each genome ranged from 1 to 157 (Figure 2B). When the core genes were classified according to their function, the major functional category identified was associated with the utilization of host-derived glycans. These genes allow B. bifidum to break down host-derived glycans, such as human milk oligosaccharides and mucin, and they are closely related to the frequent localization of B. bifidum in the intestinal tract of infants. Another common functional category was associated with gene transcriptional regulators. B. bifidum was also found to harbor two core genes encoding an ABC-type dipeptide transport system, which is related to the quorum-sensing pathway.
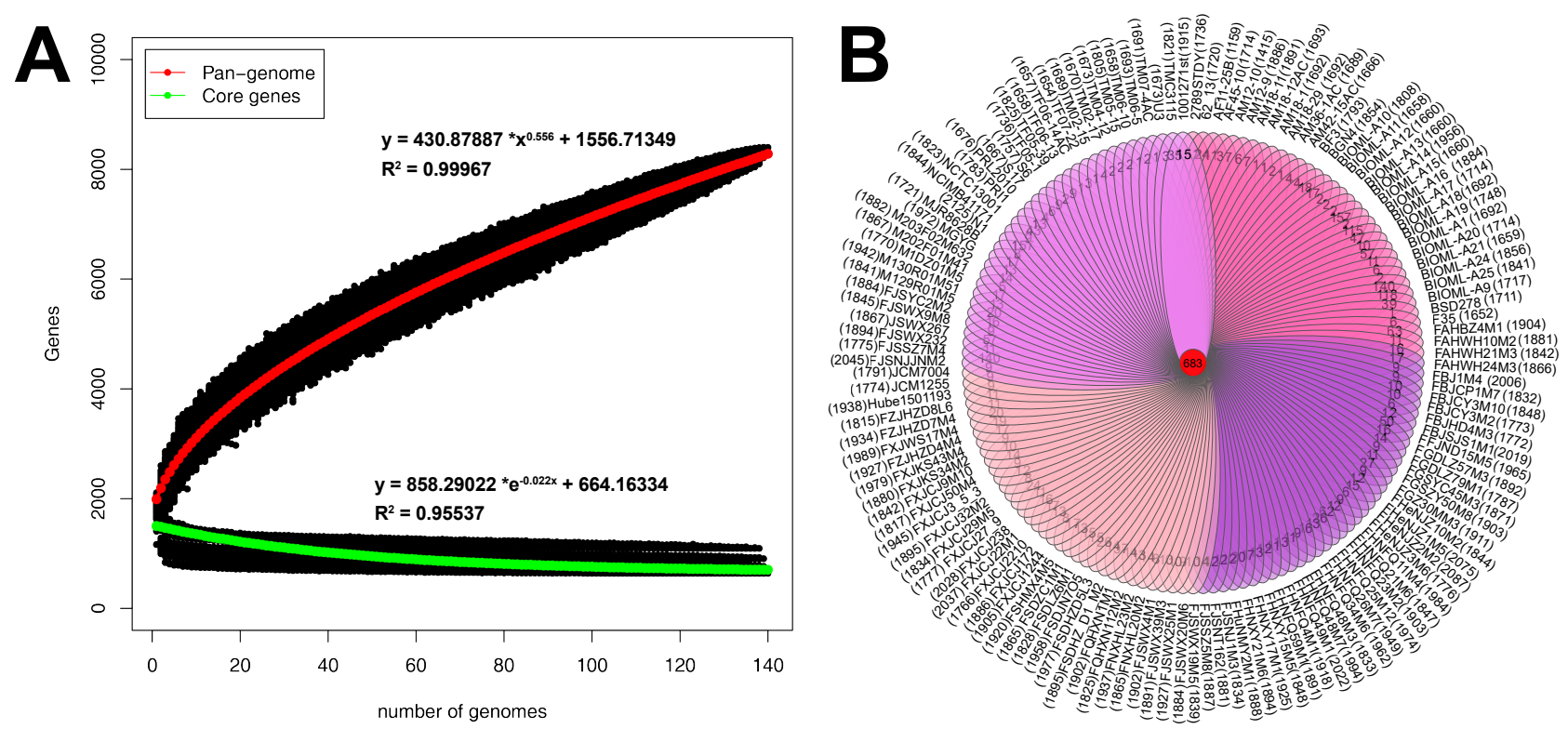

Figure 2. Pan-genome and core genes of 140 B. bifidum genomes (A). The 683 core genes and unique genes among B. bifidum genomes $(\mathbf{B})$.

Furthermore, to evaluate the contribution of the genomes sequenced in this study to the genetic diversity of B. bifidum, we performed the same analysis on $55 \mathrm{~B}$. bifidum reference genomes. This analysis showed that there were 4602 genes in the pan-genome, 669 genes in the core genome (Figure S1A), and 707 homologous genes (Figure S1B). Based on the results of the two analyses, the sequencing data from this study greatly increased our knowledge of the genetic diversity of B. bifidum and confirmed that B. bifidum has an open pan-genome and a conserved core genome. 


\subsection{Phylogenetic Analysis of B. bifidum}

To further explore the evolution and genetic variation of B. bifidum, we constructed a phylogenetic tree based on the homologous genes of 140 B. bifidum genomes (Figure 3). Based on the root branches of the tree and their bootstrap values, 126 of the 140 strains were divided into eight distinct clusters. The remaining 14 strains, except for B. bifidum M203F02M632, were grouped into several small clusters containing two to five individual strains. An analysis by geography showed that the strains located in clusters A, C, D, E, and $\mathrm{F}$ were all isolated from China. The strains located in clusters $\mathrm{A}, \mathrm{C}$, and $\mathrm{E}$ were all isolated in this study, indicating that these newly isolated strains have greatly expanded the known genetic diversity of $B$. bifidum. Furthermore, all isolates within cluster $\mathrm{G}$ were isolated in Western countries. These findings indicate that the phylogeny of B. bifidum may be related to the geographical features of its isolation source and the number of strain-specific genes has a certain relationship with the niche in which they are located.

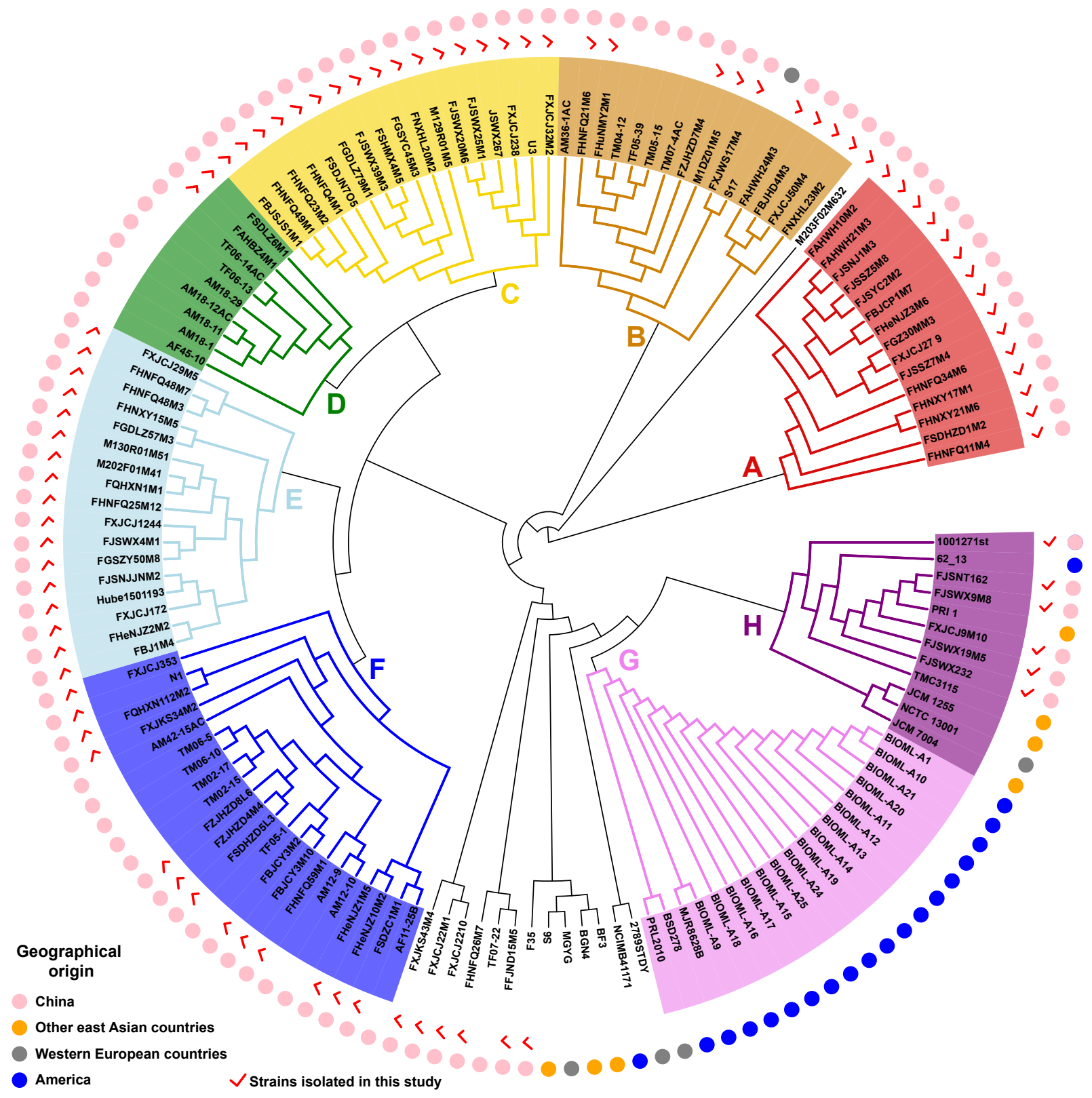

Figure 3. The phylogenetic tree of 140 B. bifidum genomes based on orthologous genes. 


\subsection{Identification of CRISPR-Cas Systems and Prophages in B. bifidum}

The CRISPR-Cas system confers adaptive immunity to bacteria to resist the insertion of foreign genes, and this system has been gradually implemented for gene editing in bacteria [43]. To provide insights into the diversity of the CRISPR-Cas system in B. bifidum, we identified CRISPR-Cas loci and characterized the architecture of each subtype. Overall, $40 \%(56 / 140)$ of the B. bifidum genomes encoded CRISPR-Cas systems (Table S2), with type II systems $(27 \%)$ being more prevalent than type I systems (13\%). Based on the presence of signature Cas proteins, we identified 36 strains with a type IIA system, and two strains with a type IIC system. All type I-positive strains had the IC system subtype. Notably, this is the first time that the type I system has been identified in B. bifidum. Phylogenetic analysis based on direct repeat sequences was then performed to classify the CRISPR-Cas systems in B. bifidum in more detail (Figure 4A). The resulting phylogenetic tree had five major branches. Subtypes IIA and IC were represented by two different branches, whereas subtype IIC had a separate branch, suggesting that the repeat sequences of the type IC and IIA systems are variable. By predicting the secondary structures of the repeat sequences, it was found that both types/subtypes had a typical stable stem-loop structure (Figure 4B). Specifically, for type IIA_1, there were two loops located at the ends of the repeat sequence. In contrast, type IIA_2 contained one additional loop located in the middle of the structure. In addition, the number of spacers in B. bifidum was variable, ranging from 38 to 190, thus illustrating that these CRISPR-Cas systems retain immunity memory [43].

As CRISPR-Cas systems may effectively prevent the integration of prophages [44], the 140 B. bifidum genomes were scanned for prophage identification. Overall, 215 prophage regions were identified in 136 genomes (Table S3 and Figure 4C); however, none of these were classified as an "intact prophage." These prophage fragments were incomplete and did not encode functional forms, indicating that functional prophages were virtually absent from B. bifidum. Moreover, there was no significant difference in the number of prophage fragments carried by CRISPR-positive and -negative strains (Figure S2). Although the repeat sequence was usually highly conserved throughout the locus, polymorphisms could be observed, notably for the terminal repeat.

\subsection{Distribution of Bacteriocin Operons in B. bifidum}

Bacteriocin is an antimicrobial peptide produced by bacteria. Bacteriocin-producing lactic acid bacteria can effectively inhibit the growth and reproduction of competing bacteria and thus occupy a dominant niche [45]. In this study, only nine potential bacteriocin operons were identified in five genomes (Figure 5). B. bifidum synthesizes four bacteriocins, including flavucin, propionicin, $\mathrm{N}$-acetylimidazole, and geobacillin, which belong to class I or II. However, four of the nine operons appeared to be incomplete because they did not contain specific ATP-binding cassette transporters. Additionally, the abundance of $B$. bifidum in the human gut gradually decreases with the age of the host [1], and these five bacteriocin-positive B. bifidum strains were isolated from adult feces. Therefore, we speculated that $B$. bifidum may enhance its ecological adaptability by producing bacteriocins.

\subsection{Carbohydrate Utilization Capacity and Genotype Binding Analysis}

As previously mentioned, several B. bifidum strains have been reported to utilize host-derived glycans. To determine whether this special carbohydrate utilization capacity is ubiquitous across the species, a local BLAST alignment, with B. bifidum PRL2010 as the reference, was performed to identify homologous genes associated with mucin utilization in the 85 isolated strains. As shown in Figure S3, all genes related to mucin metabolism, including those encoding $\beta$-N-acetylhexosaminidase, 1,2-A-L-fucosidase, $\alpha-1,3 / 4$-fucosidase, $1,3-\beta$-galactosyl- $\mathrm{N}$-acetylhexosamine phosphorylase, endo- $\alpha-\mathrm{N}$-acetylgalactosaminidase, $\beta$ galactosidase, lacto-N-biosidase, and exo- $\alpha$-sialidase, were present in all 85 B. bifidum genomes.

To verify the genotypes and phenotypes of $B$. bifidum strains that utilized host-derived and plant-derived glycans, different carbon sources were tested individually. As shown in Figure 6A, all tested strains utilized three host-derived glycogens (lactose, 2' -fucosyllactose, 
and mucin). In contrast, only a few strains utilized fructo-oligosaccharide and sucrose, and none of the tested strains utilized inulin or soluble starch. These results indicated that $B$. bifidum has the universal capacity to utilize host-derived glycogens, but it does not utilize plant-derived glycogens.
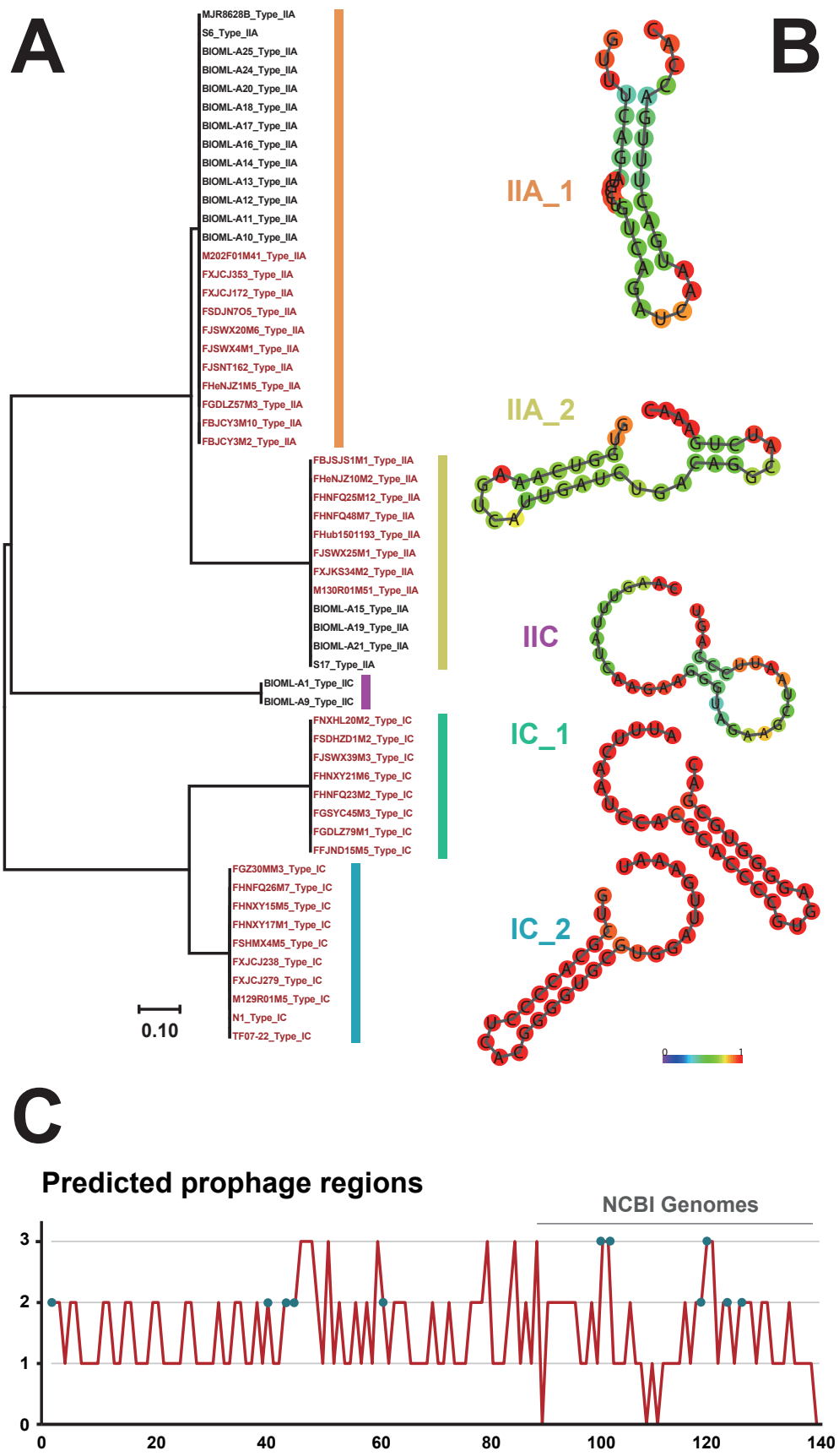

Figure 4. The phylogenetic tree based on direct repeat sequences (A), the color bars represent different subtypes of CRISPR-Cas systems in B. bifidum genomes. The secondary structures of the repeat sequences in $B$. bifidum strains (B), the color in each circle represents the frequency of the amino acid with a gradient from blue (low) to red (high). The prophage regions were identified in $B$. bifidum strains (C).

In silico predictions of CAZys were performed for the 85 B. bifidum genomes to explain the difference in glycogen utilization by B. bifidum strains from different sources. Fifty-two types of CAZys were identified, including 28 GHs, 11 GTs, 7 CEs, and 6 CBMs, and 29 types of CAZys were present in more than $90 \%$ of the strains tested (Figure 6B). The ubiquitous 
GH95 family of genes ( $\alpha$-1,2-L-fucosidase [EC 3.2.1.63] and $\alpha$-L-galactosidase [EC 3.2.1.22]) in B. bifidum explained its phenotype of lactose/2'-fucosyllactose utilization. Notably, we identified GH32 family genes (fructosyltransferases related to fructan and sucrose) in the nine strains that utilized sucrose and fructo-oligosaccharide, while this family of genes was not present in other B. bifidum strains. Additionally, these nine strains carried GH51 (endo- $\beta$-1,4-xylanase [EC 3.2.1.8], $\beta$-xylosidase [EC 3.2.1.37], $\alpha$-L-arabinofuranosidase [EC 3.2.1.55]) but lacked GH84 (N-acetyl $\beta$-glucosaminidase [EC 3.2.1.52]), whereas the other 76 B. bifidum strains carried GH84, but lacked GH51. Therefore, these strains have the potential to utilize other plant-derived glycans. Upon tracing the isolation sources of these nine strains, it was found that they were all isolated from adult feces. Therefore, we propose that the ability of $B$. bifidum to use carbohydrates was strain-specific. Some $B$. bifidum strains have developed the capacity to metabolize plant-derived glycogens to adapt to the adult intestinal environment.
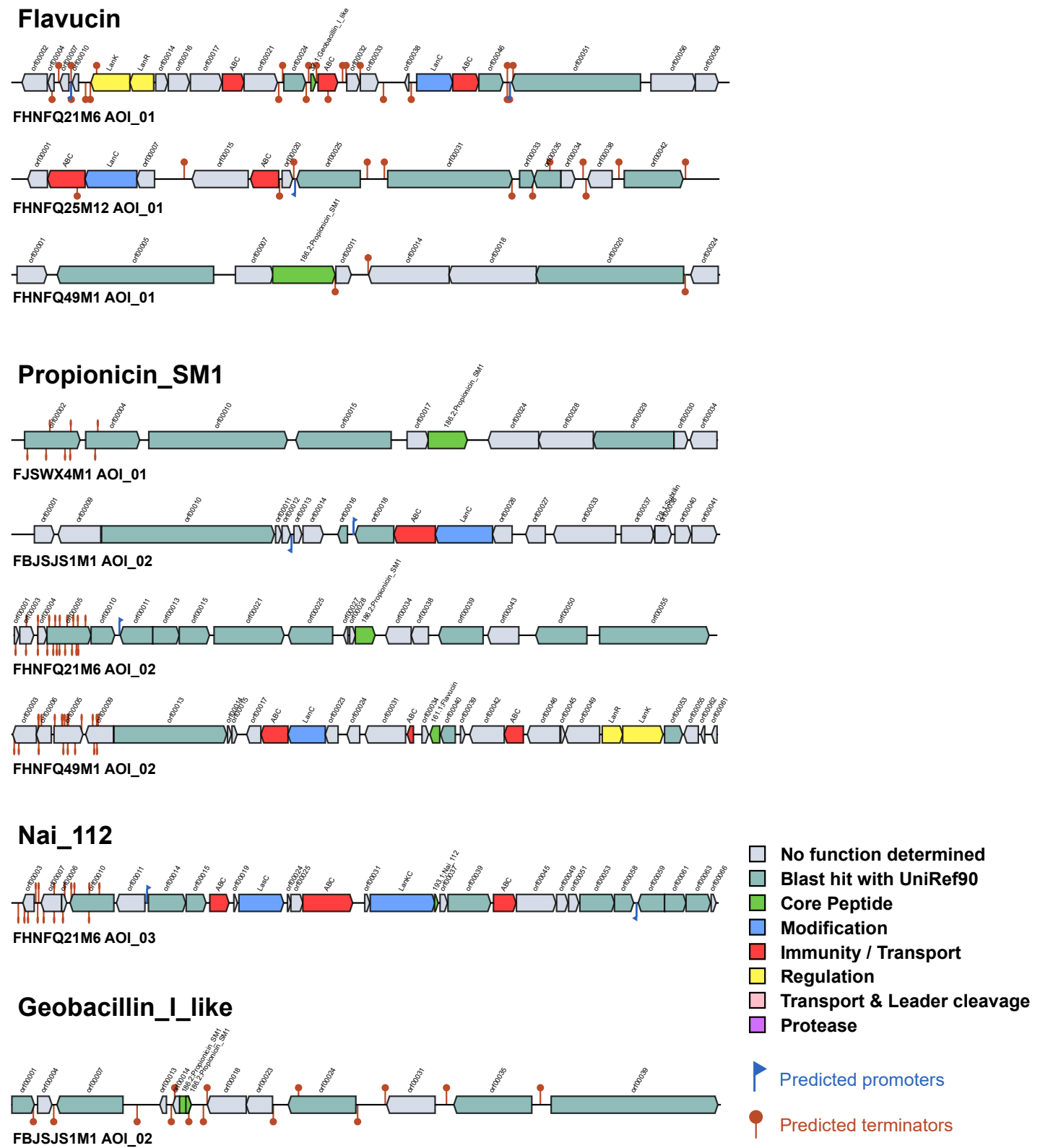

Figure 5. The potential bacteriocin operons identified in five B. bifidum genomes. 


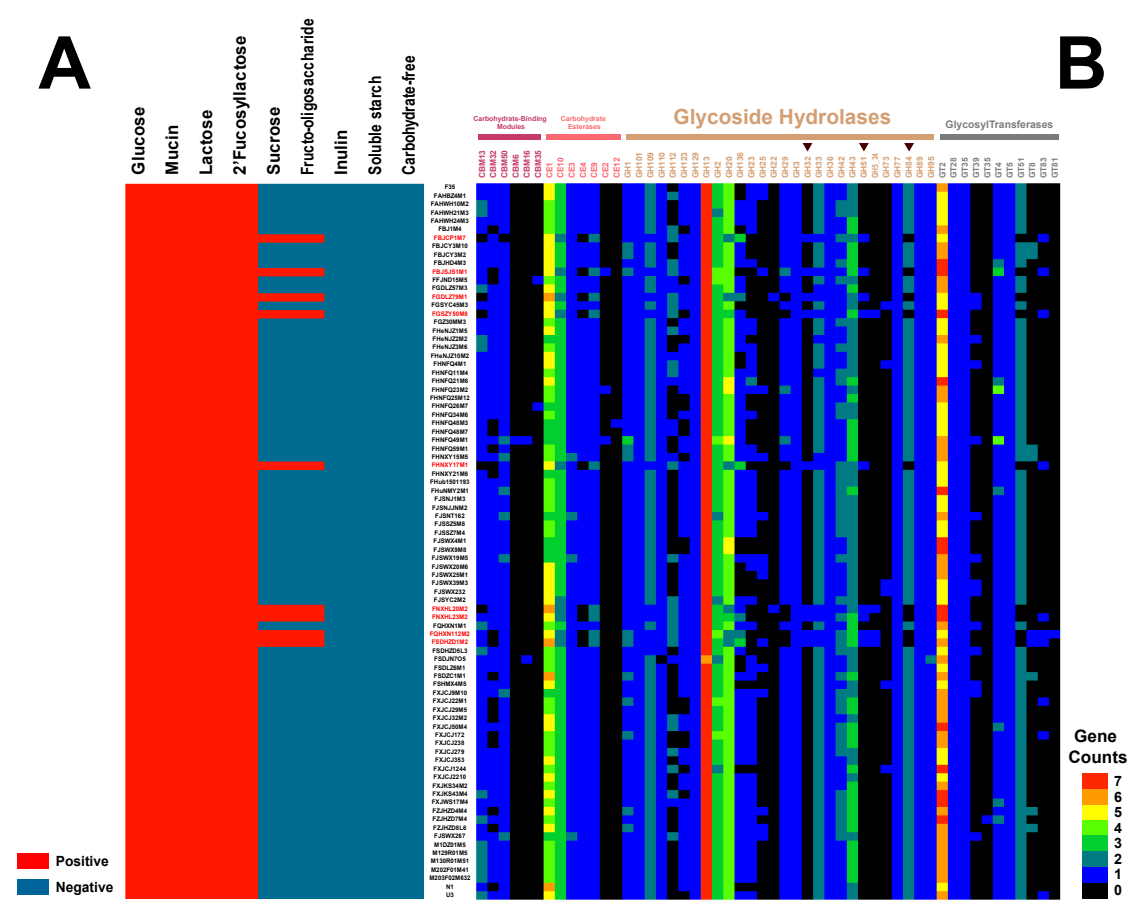

Figure 6. The phenotypes of B. bifidum strains that utilized host-derived and plant-derived glycans (A). The distribution and number of glycoside hydrolase (GH), carbohydrate esterase (CE), and glycosyltransferase (GT) family genes. Gene copy number was indicated by color ranging from dark (absent) to red (B).

\subsection{Antibiotic Resistance Genotype and Phenotype Analyses of B. bifidum}

To evaluate the safety of $B$. bifidum, based on antibiotic resistance, and to provide a reference for future applications in the probiotics industry, $140 \mathrm{~B}$. bifidum genomes were scanned to determine whether they harbored potential ARGs. As shown in Figure 7A, each strain carried an average of 106 ARGs, belonging to 129 different types. Fifty-eight of these ARG types, covering a range of common clinically used antibiotics, such as macrolide, tetracycline, fluoroquinolone, rifamycin, nitroimidazole, lincosamide, fosfomycin, and (glyco) peptide, were carried by more than $90 \%$ of the genomes tested. Of the remaining 81 types of ARGs, 57 were present in less than $10 \%$ of the strains tested, whereas the other 24 types were unevenly distributed in B. bifidum strains.

We then measured the minimum inhibitory concentration (MIC) values of 10 antibiotics for the 85 isolated B. bifidum strains (Table S4). The proportions of strains with different MIC values are shown in Figure 7B. Based on the breakpoint values of six antibiotics for the genus Bifidobacterium, as recommended by the European Food Safety Authority, most of the B. bifidum strains were found to be sensitive to ampicillin, chloramphenicol, and vancomycin. Twenty-nine strains were resistant to either erythromycin or clindamycin, and 27 of these strains were resistant to both antibiotics. This indicated that a substantial proportion of $B$. bifidum strains have developed co-resistance to erythromycin and clindamycin. Similarly, approximately $30 \%$ of the strains showed tetracycline resistance. For the other four antibiotics without any reference values, analyses of the distribution ranges of MIC values indicated that $95 \%$ of the strains (82/85) were sensitive to amoxicillin and rifampicin. Trimethoprim and ciprofloxacin were the two antibiotics with the most variability, with different strains showing different sensitivities to these antibiotics. The MIC values of these antibiotics varied from the lowest concentration to the highest. 


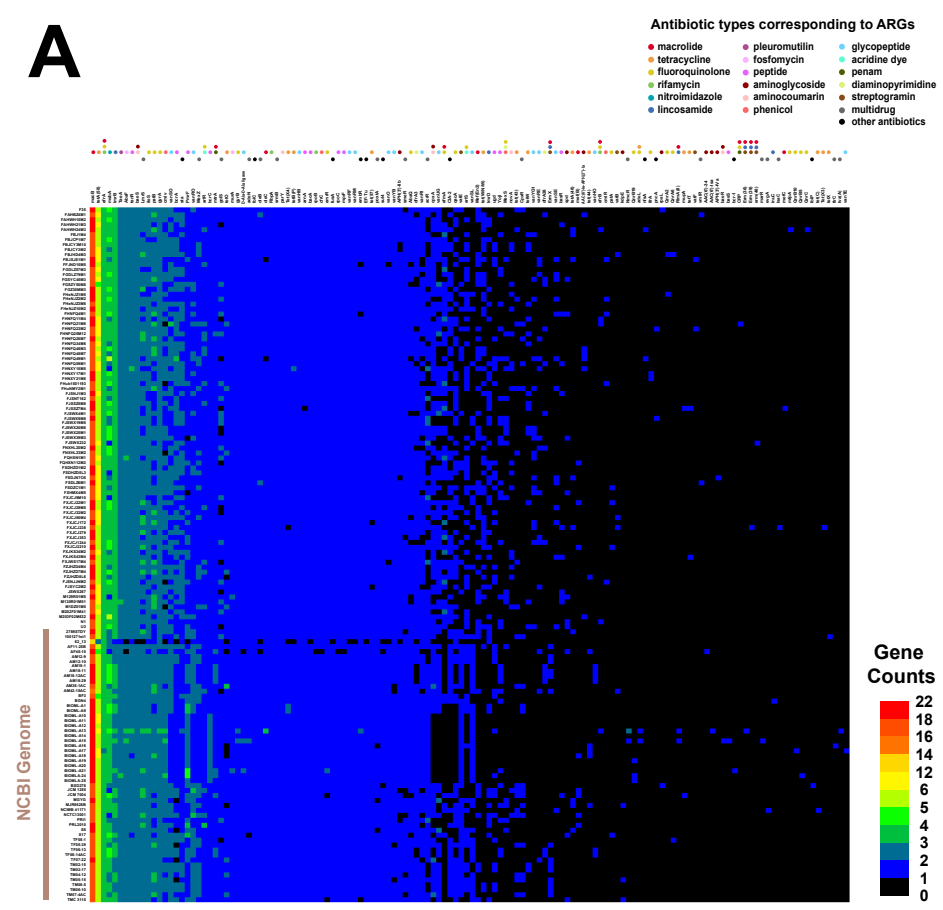

\section{B}
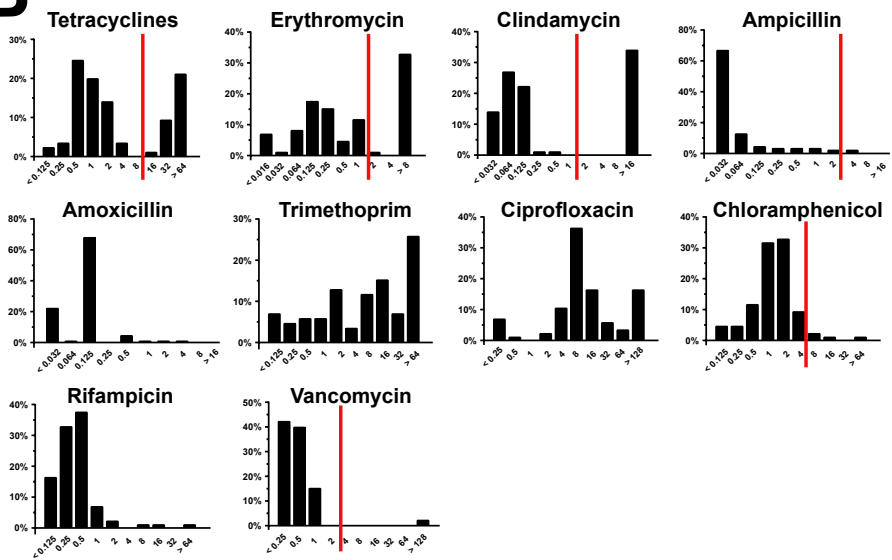

Figure 7. The distribution and the number of potential ARGs in 140 B. bifidum genomes. Gene copy number was indicated by color ranging from dark (absent) to red (A). The proportions of strains with different MIC values (B). The red lines represent microbiological breakpoints recommended by the EFSA.

\section{Discussion}

Among members of the genus Bifidobacterium, B. adolescentis, B. animals, B. dentium, and B. pseudolongum are considered to be cosmopolitan species, whereas B. bifidum displays a more singular lifestyle and has been specifically identified in the gut of breast-fed infants. In this context, previous comparative genomics studies have concluded that B. bifidum is a relatively conservative species, with limited niche adaptability, low genetic diversity, and an open pan-genome. However, the development of genomic tools and the increased number of available sequences have facilitated further analyses of the genomic diversity and function of B. bifidum. In this study, genomes of 85 strains isolated from the feces of Chinese infants and adults, combined with 55 publicly available genomes, were used to perform a more comprehensive comparative genomic analysis. We found that B. bifidum is not conservative, as previously reported, and a potential mechanism for its adaptation to the adult intestinal environment may exist in some strains. Moreover, new insights into 
phylogenetic correlations, mobile genetic elements, and antibiotic resistance of B. bifidum from this study expand our knowledge of this species.

The average genome size of the 140 B. bifidum strains was $2.17 \mathrm{Mb}$, which is consistent with previous reports [13]. Our genome-wide study of B. bifidum showed a trend of the gradual opening up of the genome, suggesting that $B$. bifidum has an open pan-genome and that its pan-genome will increase if more genomes are analyzed and more novel gene families are identified. Moreover, a more open pan-genome implies that gene exchange is higher within the species. These data support the hypothesis that the relative size and content of the pan-genome are potential indicators of the genetic plasticity and environmental adaptation potential of the species. In addition, by annotating the core genes, the defense mechanisms and general functions of the species were predicted.

CRISPR loci, which are present in the genomes of a large number of lactic acid bacteria, provide acquired immunity against foreign genetic elements [44]. However, few research studies have focused on CRISPR in B. bifidum. In this study, we specifically analyzed the CRISPR-Cas system in all 140 strains of B. bifidum and found that $40 \%$ of the strains contained a complete system. This implied that B. bifidum is a good candidate for gene editing and the cleavage of lytic bacteriophages in the food industry. The incomplete CRISPR-Cas loci in the remaining strains may be due to genetic recombination, loss of the ability to acquire other CRISPR loci, or the incomplete assembly of the genomes of these strains. The diversity of the Cas protein was significant, but the CRISPR-Cas system could be easily classified (type I-III) by identifying the characteristic proteins (Cas3, Cas9, and Cas10) encoded in the genomes. We found that the type IC, type IIA, and type IIC systems were widespread across B. bifidum strains. It is worth noting that the type I system was detected for the first time in B. bifidum in this study and was present in $13 \%$ of the strains tested. These data provide a new perspective for future investigations of genome diversity and the CRISPR-Cas system in B. bifidum.

Screening for bacteriocin in vitro is complex and difficult to achieve, whereas in silico analysis using BAGEL to identify potential bacteriocin operons is generally a more feasible technique. In this study, only nine potential bacteriocin operons, including flavucin, propionicin SM1, Nai_112, and geobacillin_I, were identified in five B. bifidum genomes. There has been little research on bacteriocins produced by B. bifidum. B. bifidum NCFB 1454 has been reported to have the ability to produce bifidocin B [46]. Flavucin is produced by Corynebacterium lipophiloflavum DSM 44,291 and displays high antimicrobial activity [47]. Propionicin SM1 is a bacteriocin isolated from Propionibacterium jensenii DF1 [48]. Nai_112 is a glycosylated class III lanthipeptide produced by an Actinoplanes sp. strain with potent bioactivity against nociceptive pain [49]. Geobacillin I is isolated from the thermophilic bacterium Geobacillus thermodenitrificans NG80-2 [50]. The presence of bacteriocin facilitates survival in complex environments and provides bacteria with a competitive advantage [51]. Bacteriocin production may confer B. bifidum with a competitive advantage in the intestine and increase its potential for use as a probiotic.

Through direct regulation or indirect modulation via the host microbiota, B. bifidum plays an impressive role in attenuating both GI diseases and diseases in remote tissues [12]. There are multiple B. bifidum strains with different host origins, and many of the probiotic functions of $B$. bifidum are strain-dependent. Therefore, it may be advantageous to combine different strains of B. bifidum to maximize their beneficial effects. However, caution should be taken when drawing inferences based on in silico predictions. Thus, future in vivo analyses performed in murine models or clinical trials will be needed to validate the results described in this study.

\section{Conclusions}

A comparative genomic analysis of 140 strains of B. bifidum isolated from different niches was performed in this study. The comparative genomic results show that the number of strain-specific genes has a certain relationship with the niche in which they are located. The core functions of B. bifidum were concentrated on the utilization of host- 
derived glyans, which provide molecular support for strains to metabolize multiple sugars. Further literature mining of the carbohydrate metabolism-related genes would improve the prediction accuracy of this method. Additionally, the genetic diversity of B. bifidum was mainly reflected in the glycoside hydrolase gene family, bacteriocin operons, antibiotic resistance genes, and CRISPR-Cas loci. These results provide new information and a framework for further investigating the evolution.

Supplementary Materials: The following are available online at https://www.mdpi.com/article/10 $.3390 /$ genes12101504/s1, Figure S1: Pan-genome, core genes and unique genes among 55 B. bifidum genomes accessed from NCBI, Figure S2: Comparison of the number of prophage fragments in $B$. bifidum genomes with or without a CRISPR-Cas system, Figure S3: Homologous genes associated with mucin utilization in the 85 B. bifidum isolated strains, Table S1: Specific information of $140 \mathrm{~B}$. bifidum genomes involved in this study, Table S2: The distribution of CRISPR-Cas systems among 140 B. bifidum, Table S3: Prediction of prophages among 140 B. bifidum genomes, Table S4: The minimum inhibitory concentration of 85 B. Bifidum to ten antibiotics.

Author Contributions: Conceptualization: H.Z. and H.W.; Methodology, H.W. and W.L.; Software, H.W. and Z.P.; Validation, J.Z., W.C. and H.Z.; Formal Analysis, H.W., W.L. and M.Z.; Investigation, H.W., W.L. and M.Z.; Resources, J.Z., W.C. and H.Z.; Data Curation, H.W. and Z.P.; Writing-Original Draft Preparation, H.W., W.L. and Z.P.; Writing-Review \& Editing, Y.-k.L.; Visualization, W.L. and Z.P.; Supervision, Y.-k.L., H.Z.; Project Administration, H.Z.; Funding Acquisition, J.Z., W.C. and H.Z. All authors have read and agreed to the published version of the manuscript.

Funding: The research was funded by the National Nature Science Foundation of China (31771953, 31820103010), the Fundamental Research Funds for the Central Universities (JUSRP51903B), 111project (BP0719028), the International Cooperation Fund Pool Project of Jiangsu Industrial Technology Research Institute, the collaborative innovation center of food safety and quality control in Jiangsu Province, and the National First-class Discipline Program of Food Science and Technology (JUFSTR20180102).

Institutional Review Board Statement: Not applicable.

Informed Consent Statement: Not applicable.

Data Availability Statement: This study generated sequencing data for 85 B. bifidum isolates, and all sequence data have been deposited in the National Center for Biotechnology Information.

Conflicts of Interest: Authors have no conflict of interest to disclose.

\section{References}

1. Turroni, F.; Duranti, S.; Milani, C.; Lugli, G.A.; van Sinderen, D.; Ventura, M. Bifidobacterium bifidum: A key member of the early human gut microbiota. Microorganisms 2019, 7, 544. [CrossRef]

2. Turroni, F.; Duranti, S.; Bottacini, F.; Guglielmetti, S.; Van Sinderen, D.; Ventura, M. Bifidobacterium bifidum as an example of a specialized human gut commensal. Front. Microbiol 2014, 5, 437. [CrossRef]

3. Stewart, C.J.; Ajami, N.J.; O’Brien, J.L.; Hutchinson, D.S.; Smith, D.P.; Wong, M.C.; Ross, M.C.; Lloyd, R.E.; Doddapaneni, H.; Metcalf, G.A.; et al. Temporal development of the gut microbiome in early childhood from the TEDDY study. Nature 2018, 562, 583-588. [CrossRef]

4. Yamamoto, K. Biological analysis of the microbial metabolism of hetero-oligosaccharides in application to glycotechnology. Biosci. Biotechnol. Biochem. 2012, 76, 1815-1827. [CrossRef]

5. Turroni, F.; Bottacini, F.; Foroni, E.; Mulder, I.; Kim, J.H.; Zomer, A.; Sanchez, B.; Bidossi, A.; Ferrarini, A.; Giubellini, V.; et al. Genome analysis of Bifidobacterium bifidum PRL2010 reveals metabolic pathways for host-derived glycan foraging. Proc. Natl. Acad. Sci. USA 2010, 107, 19514-19519. [CrossRef] [PubMed]

6. Turroni, F.; Milani, C.; van Sinderen, D.; Ventura, M. Genetic strategies for mucin metabolism in Bifidobacterium bifidum PRL2010: An example of possible human-microbe co-evolution. Gut Microbes 2011, 2, 183-189. [CrossRef] [PubMed]

7. Khailova, L.; Mount Patrick, S.K.; Arganbright, K.M.; Halpern, M.D.; Kinouchi, T.; Dvorak, B. Bifidobacterium bifidum reduces apoptosis in the intestinal epithelium in necrotizing enterocolitis. Am. J. Physiol. Gastrointest. Liver Physiol. 2010, 299, G1118-G1127. [CrossRef] [PubMed]

8. Wang, Q.; Wang, K.; Wu, W.; Lv, L.; Bian, X.; Yang, L.; Wang, Q.; Li, Y.; Ye, J.; Fang, D.; et al. Administration of Bifidobacterium bifidum CGMCC 15068 modulates gut microbiota and metabolome in azoxymethane (AOM)/dextran sulphate sodium (DSS)induced colitis-associated colon cancer (CAC) in mice. Appl. Microbiol. Biotechnol. 2020, 104, 5915-5928. [CrossRef] 
9. Duranti, S.; Gaiani, F.; Mancabelli, L.; Milani, C.; Grandi, A.; Bolchi, A.; Santoni, A.; Lugli, G.A.; Ferrario, C.; Mangifesta, M.; et al. Elucidating the gut microbiome of ulcerative colitis: Bifidobacteria as novel microbial biomarkers. FEMS Microbiol. Ecol. 2016, 92, fiw191. [CrossRef] [PubMed]

10. Zanotti, I.; Turroni, F.; Piemontese, A.; Mancabelli, L.; Milani, C.; Viappiani, A.; Prevedini, G.; Sanchez, B.; Margolles, A.; Elviri, L.; et al. Evidence for cholesterol-lowering activity by Bifidobacterium bifidum PRL2010 through gut microbiota modulation. Appl. Microbiol. Biotechnol. 2015, 99, 6813-6829. [CrossRef] [PubMed]

11. Wang, F.; Xu, T.; Zhang, Y.; Zheng, T.; He, Y.; He, F.; Jiang, Y. Long-term combined administration of Bifidobacterium bifidum TMC3115 and Lactobacillus plantarum 45 alleviates spatial memory impairment and gut dysbiosis in APP/PS1 mice. FEMS Microbiol. Lett. 2020, 367, fnaa048. [CrossRef] [PubMed]

12. Verma, R.; Lee, C.; Jeun, E.J.; Yi, J.; Kim, K.S.; Ghosh, A.; Byun, S.; Lee, C.G.; Kang, H.J.; Kim, G.C.; et al. Cell surface polysaccharides of Bifidobacterium bifidum induce the generation of Foxp3(+) regulatory T cells. Sci. Immunol. 2018, 3. [CrossRef]

13. Duranti, S.; Milani, C.; Lugli, G.A.; Turroni, F.; Mancabelli, L.; Sanchez, B.; Ferrario, C.; Viappiani, A.; Mangifesta, M.; Mancino, W.; et al. Insights from genomes of representatives of the human gut commensal Bifidobacterium bifidum. Environ. Microbiol 2015, 17, 2515-2531. [CrossRef] [PubMed]

14. Yu, D.S.; Jeong, H.; Lee, D.H.; Kwon, S.K.; Song, J.Y.; Kim, B.K.; Park, M.S.; Ji, G.E.; Oh, T.K.; Kim, J.F. Complete genome sequence of the probiotic bacterium Bifidobacterium bifidum strain BGN4. J. Bacteriol. 2012, 194, 4757-4758. [CrossRef]

15. Zhurina, D.; Zomer, A.; Gleinser, M.; Brancaccio, V.F.; Auchter, M.; Waidmann, M.S.; Westermann, C.; van Sinderen, D.; Riedel, C.U. Complete genome sequence of Bifidobacterium bifidum S17. J. Bacteriol. 2011, 193, 301-302. [CrossRef]

16. Gueimonde, M.; Ventura, M.; Margolles, A.; Sanchez, B. Genome sequence of the immunomodulatory strain Bifidobacterium bifidum LMG 13195. J. Bacteriol. 2012, 194, 6997. [CrossRef]

17. Andryuschenko, S.V.; Ivanova, E.V.; Perunova, N.B.; Zdvizhkova, I.A.; Bekpergenova, A.V.; Bukharin, O.V. Draft genome sequence of Bifidobacterium bifidum strain ICIS-310, isolated from the feces of a healthy 5-year-old child from Orenburg, Russia. Microbiol. Resour. Announc. 2018, 7, e01271-18. [CrossRef]

18. Albert, K.; Rani, A.; Sela, D.A. Comparative pangenomics of the mammalian gut Commensal Bifidobacterium longum. Microorganisms 2019, 8, 7. [CrossRef]

19. Lugli, G.A.; Mancino, W.; Milani, C.; Duranti, S.; Mancabelli, L.; Napoli, S.; Mangifesta, M.; Viappiani, A.; Anzalone, R.; Longhi, G.; et al. Dissecting the evolutionary development of the species Bifidobacterium animalis through comparative genomics analyses. Appl. Environ. Microbiol. 2019, 85, e02806-18. [CrossRef] [PubMed]

20. Duranti, S.; Milani, C.; Lugli, G.A.; Mancabelli, L.; Turroni, F.; Ferrario, C.; Mangifesta, M.; Viappiani, A.; Sanchez, B.; Margolles, A.; et al. Evaluation of genetic diversity among strains of the human gut commensal Bifidobacterium adolescentis. Sci. Rep. 2016, 6. [CrossRef]

21. Bottacini, F.; Morrissey, R.; Roberts, R.J.; James, K.; van Breen, J.; Egan, M.; Lambert, J.; van Limpt, K.; Knol, J.; Motherway, M.O.; et al. Comparative genome and methylome analysis reveals restriction/modification system diversity in the gut commensal Bifidobacterium breve. Nucleic Acids Res. 2018, 46, 1860-1877. [CrossRef]

22. Toshimitsu, T.; Nakamura, M.; Ikegami, S.; Terahara, M.; Itou, H. Strain-specific identification of Bifidobacterium bifidum OLB6378 by PCR. Biosci. Biotechnol. Biochem. 2013, 77, 572-576. [CrossRef]

23. Deletoile, A.; Passet, V.; Aires, J.; Chambaud, I.; Butel, M.J.; Smokvina, T.; Brisse, S. Species delineation and clonal diversity in four Bifidobacterium species as revealed by multilocus sequencing. Res. Microbiol. 2010, 161, 82-90. [CrossRef] [PubMed]

24. Xie, Y.; Wu, G.; Tang, J.; Luo, R.; Patterson, J.; Liu, S.; Huang, W.; He, G.; Gu, S.; Li, S.; et al. SOAPdenovo-Trans: De novo transcriptome assembly with short RNA-Seq reads. Bioinformatics 2014, 30, 1660-1666. [CrossRef] [PubMed]

25. Xu, M.; Guo, L.; Gu, S.; Wang, O.; Zhang, R.; Peters, B.A.; Fan, G.; Liu, X.; Xu, X.; Deng, L.; et al. TGS-GapCloser: A fast and accurate gap closer for large genomes with low coverage of error-prone long reads. Gigascience 2020, 9, giaa094. [CrossRef] [PubMed]

26. Parks, D.H.; Imelfort, M.; Skennerton, C.T.; Hugenholtz, P.; Tyson, G.W. CheckM: Assessing the quality of microbial genomes recovered from isolates, single cells, and metagenomes. Genome Res. 2015, 25, 1043-1055. [CrossRef] [PubMed]

27. Delcher, A.L.; Bratke, K.A.; Powers, E.C.; Salzberg, S.L. Identifying bacterial genes and endosymbiont DNA with Glimmer. Bioinformatics 2007, 23, 673-679. [CrossRef] [PubMed]

28. Zhao, Y.; Wu, J.; Yang, J.; Sun, S.; Xiao, J.; Yu, J. PGAP: Pan-genomes analysis pipeline. Bioinformatics 2012, 28, 416-418. [CrossRef]

29. Li, L.; Stoeckert, C.J., Jr.; Roos, D.S. OrthoMCL: Identification of ortholog groups for eukaryotic genomes. Genome Res. 2003, 13, 2178-2189. [CrossRef]

30. Katoh, K.; Standley, D.M. MAFFT: Iterative refinement and additional methods. Methods Mol. Biol. 2014, 1079, 131-146. [CrossRef]

31. Subramanian, B.; Gao, S.; Lercher, M.J.; Hu, S.; Chen, W.H. Evolview v3: A webserver for visualization, annotation, and management of phylogenetic trees. Nucleic Acids Res. 2019, 47, W270-W275. [CrossRef]

32. Couvin, D.; Bernheim, A.; Toffano-Nioche, C.; Touchon, M.; Michalik, J.; Neron, B.; Rocha, E.P.C.; Vergnaud, G.; Gautheret, D.; Pourcel, C. CRISPRCasFinder, an update of CRISRFinder, includes a portable version, enhanced performance and integrates search for Cas proteins. Nucleic Acids Res. 2018, 46, W246-W251. [CrossRef]

33. Abby, S.S.; Neron, B.; Menager, H.; Touchon, M.; Rocha, E.P. MacSyFinder: A program to mine genomes for molecular systems with an application to CRISPR-Cas systems. PLoS ONE 2014, 9, e110726. [CrossRef] 
34. Gruber, A.R.; Lorenz, R.; Bernhart, S.H.; Neuböck, R.; Hofacker, I.L. The Vienna RNA Websuite. Nucleic Acids Res. 2008, 36, W70-W74. [CrossRef] [PubMed]

35. Kumar, S.; Stecher, G.; Li, M.; Knyaz, C.; Tamura, K. MEGA X: Molecular evolutionary genetics analysis across computing platforms. Mol. Biol. Evol. 2018, 35, 1547-1549. [CrossRef] [PubMed]

36. Arndt, D.; Marcu, A.; Liang, Y.; Wishart, D.S. PHAST, PHASTER and PHASTEST: Tools for finding prophage in bacterial genomes Brief. Bioinform. 2019, 20, 1560-1567. [CrossRef] [PubMed]

37. Van Heel, A.J.; de Jong, A.; Song, C.; Viel, J.H.; Kok, J.; Kuipers, O.P. BAGEL4: A user-friendly web server to thoroughly mine RiPPs and bacteriocins. Nucleic Acids Res. 2018, 46, W278-W281. [CrossRef] [PubMed]

38. Lombard, V.; Golaconda Ramulu, H.; Drula, E.; Coutinho, P.M.; Henrissat, B. The carbohydrate-active enzymes database (CAZy) in 2013. Nucleic Acids Res. 2014, 42, D490-D495. [CrossRef]

39. Alcock, B.P.; Raphenya, A.R.; Lau, T.T.Y.; Tsang, K.K.; Bouchard, M.; Edalatmand, A.; Huynh, W.; Nguyen, A.V.; Cheng, A.A.; Liu, S.; et al. CARD 2020: Antibiotic resistome surveillance with the comprehensive antibiotic resistance database. Nucleic Acids Res. 2020, 48, D517-D525. [CrossRef]

40. International Organization for Standardization (ISO). Milk and Milk Products: Determination of the Minimal Inhibitory Concentration (MIC) of Antibiotics Applicable to Bifidobacteria and Non-Enterococcal Lactic Acid Bacteria (LAB); ISO 10932:2010 (IDF 223:2010); ISO: Geneva, Switzerland, 2010.

41. Deng, W.; Wang, Y.; Liu, Z.; Cheng, H.; Xue, Y. HemI: A toolkit for illustrating heatmaps. PLoS ONE 2014, 9, e111988. [CrossRef]

42. Ciufo, S.; Kannan, S.; Sharma, S.; Badretdin, A.; Clark, K.; Turner, S.; Brover, S.; Schoch, C.L.; Kimchi, A.; DiCuccio, M. Using average nucleotide identity to improve taxonomic assignments in prokaryotic genomes at the NCBI. Int. J. Syst. Evol. Microbiol. 2018, 68, 2386-2392. [CrossRef] [PubMed]

43. Hidalgo-Cantabrana, C.; Crawley, A.B.; Sanchez, B.; Barrangou, R. Characterization and exploitation of CRISPR loci in Bifidobacterium longum. Front. Microbiol. 2017, 8, 1851. [CrossRef] [PubMed]

44. Pei, Z.; Sadiq, F.A.; Han, X.; Zhao, J.; Zhang, H.; Ross, R.P.; Lu, W.; Chen, W. Comprehensive scanning of prophages in Lactobacillus: Distribution, diversity, antibiotic resistance genes, and linkages with CRISPR-Cas systems. mSystems 2021, 6, e01211-20. [CrossRef] [PubMed]

45. Yadav, M.K.; Singh, B.; Tiwari, S.K. Comparative analysis of inhibition-based and indicator-independent colorimetric assay for screening of bacteriocin-producing lactic acid bacteria. Probiotics Antimicrob. Proteins 2019, 11, 687-695. [CrossRef]

46. Yildirim, Z.; Winters, D.K.; Johnson, M.G. Purification, amino acid sequence and mode of action of bifidocin B produced by Bifidobacterium bifidum NCFB 1454. J. Appl. Microbiol. 1999, 86, 45-54. [CrossRef]

47. Van Heel, A.J.; Kloosterman, T.G.; Montalban-Lopez, M.; Deng, J.; Plat, A.; Baudu, B.; Hendriks, D.; Moll, G.N.; Kuipers, O.P. Discovery, production and modification of five novel Lantibiotics using the promiscuous Nisin modification machinery. ACS Synth. Biol. 2016, 5, 1146-1154. [CrossRef]

48. Miescher, S.; Stierli, M.P.; Teuber, M.; Meile, L. Propionicin SM1, a bacteriocin from Propionibacterium jensenii DF1: Isolation and characterization of the protein and its gene. Syst. Appl. Microbiol. 2000, 23, 174-184. [CrossRef]

49. Sheng, W.; Xu, B.; Chen, S.; Li, Y.; Liu, B.; Wang, H. Substrate tolerance of the biosynthetic enzymes of glycosylated lanthipeptide NAI-112. Org. Biomol. Chem. 2020, 18, 6095-6099. [CrossRef]

50. Garg, N.; Oman, T.J.; Andrew Wang, T.S.; De Gonzalo, C.V.; Walker, S.; van der Donk, W.A. Mode of action and structure-activity relationship studies of geobacillin I. J. Antibiot. 2014, 67, 133-136. [CrossRef]

51. Dobson, A.; Cotter, P.D.; Ross, R.P.; Hill, C. Bacteriocin production: A probiotic trait? Appl. Environ. Microbiol. 2012, 78, 1-6. [CrossRef] 